\title{
Spatiotemporal guided bullets in multimode fiber
}

\author{
M. Zitelli ${ }^{\mathrm{a}}$, M. Ferraro ${ }^{\mathrm{a}}$, F. Mangini ${ }^{\mathrm{b}}$, L. Leggio ${ }^{\mathrm{a}}$, D.S. Kharenko ${ }^{\mathrm{c}}$, A. Niang $^{\mathrm{b}}$, A. Tonello $^{\mathrm{d}}$, \\ V. Couderc ${ }^{d}$, T. Hansson ${ }^{\mathrm{e}}, \mathrm{S}$. Wabnitz ${ }^{\mathrm{a}, \mathrm{c}}$

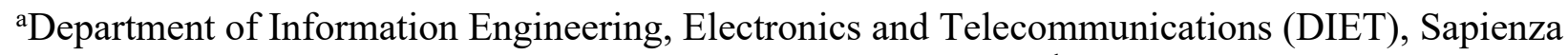 \\ University of Rome, Via Eudossiana 18, 00184 Rome, Italy; ${ }^{b}$ Department of Information \\ Engineering (DII), University of Brescia, Via Branze 38, 25123 Brescia, Italy; 'Novosibirsk State \\ University, Pirogova 1, Novosibirsk 630090, Russia; ${ }^{\mathrm{d} U n i v e r s i t e ́ ~ d e ~ L i m o g e s, ~ X L I M, ~ U M R ~ C N R S ~}$ \\ 7252, 123 Avenue A. Thomas, 87060 Limoges, France; ${ }^{e}$ Department of Physics, Chemistry and \\ Biology, Linköping University, SE-581 83 Linköping, Sweden
}

\begin{abstract}
Beam self-imaging of ultrashort pulses in nonlinear graded-index (GRIN) multimode optical fibers is of interest for many applications, including spatiotemporal mode-locking in fiber lasers. We obtained a new analytical description for the nonlinear evolution of a laser beam of arbitrary transverse shape propagating in a GRIN fiber. The longitudinal beam evolution could be directly visualized by means of femtosecond laser pulses, propagating in the anomalous or in the normal dispersion regime, leading to light scattering out of the fiber core via the emission of blue photo-luminescence. As the critical power for self-focusing is approached and even surpassed, a host of previously undisclosed nonlinear effects is revealed, including strong multiphoton absorption by oxygen-deficiency center defects and Germanium inclusions, splitting and shifting of the self-imaging period, filamentation, and conical emission of the guided light bullets. We discovered that nonlinear loss has a profound influence on the process of high-order spatiotemporal soliton fission. The beam energy carried by the fiber is clamped to a fixed value, and nonlinear bullet attractors with suppressed Raman frequency shift and fixed temporal duration are generated, leading to highly efficient frequency conversion of the input near-infrared femtosecond pulses into mid-infrared multimode solitons.
\end{abstract}

Keywords: Optical solitons, light bullets, multimode fiber, Raman scattering, self-focusing, ultrashort pulses.

\section{INTRODUCTION}

In the last decade, the study of the propagation of ultrashort pulses in multimode (MM) fibers has seen a renewed interest, thanks to the potential that this technology can offer for guided optical communications [1,2]. Despite numerous studies in the literature, several aspects are still to be fully understood.

In this work, we study the nonlinear effects due to the propagation of femtosecond pulses in different lengths of MM fiber with a graded-index (GRIN) core profile (50/125). In particular, for relatively short MM fiber lengths (i.e., $30 \mathrm{~cm}$ of GRIN fiber), we revealed a new type of high-energy pulse propagation regime, characterized by the presence of MM solitons, created by the fission of the initial femtosecond pulse [3]. We observed that these solitons have different amplitudes and wavelengths, but nearly equal time duration. Moreover, we experimentally visualized the evolution of spatial self-imaging (SSI) using ultrashort laser pulses, leading to the side-light scattering of photoluminescence and harmonic waves, confirming, indirectly, the invariance of the SSI period [4]. Finally, the evolution of spatiotemporal solitons was also experimentally investigated over relatively long (i.e., up to $1 \mathrm{~km}$ ) spans of GRIN fiber, demonstrating that the fundamental mode behaves as an attractor with respect to higher-order modes.

To study the generation and fission of high-energy MM solitons, and the longitudinal evolution of beam spatial selfimaging in the anomalous dispersion regime, we used a hybrid optical parametric amplifier generating pulses with $70 \mathrm{fs}$ duration at $1550 \mathrm{~nm}$, and a pulse repetition rate of up to $100 \mathrm{kHz}$. 


\section{BEAM SELF-IMAGING OF ULTRASHORT PULSES}

In this section, we present a derivation of the exact solution for the nonlinear evolution of first and second-order moments of a laser beam of arbitrary transverse shape in a GRIN fiber [4]. By using operator methods of quantum mechanics, we theoretically determine, in full generality, that the SSI period only varies with the fiber characteristics and the laser wavelength (but it is independent of the laser power); our method also allows for estimating the power threshold for catastrophic self-focusing in a GRIN MMF for a beam of arbitrary incidence.

Whereas, we achieved direct experimental evidence of SSI, by exciting up-conversion luminescence (UL) of fiber material defects by means of intense femtosecond laser pulse-induced multiphoton absorption (MPA). Visible violet light is radially scattered out of the fiber at the points of maximum intensity given by the SSI, allowing for a direct measurement of the SSI period.

\subsection{Theory}

We consider spatial beam propagation in a GRIN fiber with an infinite extended parabolic index profile. Neglecting Raman scattering and temporal dynamics, the beam evolution is described by a 2D-NLSE with an instantaneous Kerr nonlinearity of the form

$$
\frac{\partial A}{\partial z}-i \frac{1}{2 k_{0}}\left(\frac{\partial^{2} A}{\partial x^{2}}+\frac{\partial^{2} A}{\partial y^{2}}\right)+i \frac{k_{0} \Delta}{\rho^{2}}\left(x^{2}+y^{2}\right) A=i \frac{k_{0} n_{2}}{n_{c o}}|A|^{2} A
$$

where $k_{0}=\omega_{0} n_{c o} / c$ is the wavenumber, $\rho$ is the core radius, $\Delta=\left(n_{c o}^{2}-n_{c l}^{2}\right) / 2 n_{c o}^{2}$ is the relative index difference, and $n_{c o}\left(n_{c l}\right)$ is the core (cladding) refractive index of the fiber, respectively. The propagation equation (2.1) can be rewritten in the form:

$$
i \frac{\partial A}{\partial z}=\widehat{H} A,
$$

where $\mathrm{H}$ is a pseudo-Hamiltonian operator that reads:

$$
\widehat{H}=\frac{1}{2 k_{0}}\left({\widehat{p_{x}}}^{2}+{\widehat{p_{y}}}^{2}\right)+\beta\left(\widehat{x}^{2}+\hat{y}^{2}\right)-\gamma \hat{I}
$$

being $\beta=k_{0} \Delta / \rho^{2}, \gamma=k_{0} n_{2} / n_{c o}, \hat{x}$ and $\hat{y}$ the transverse position operators, $\widehat{p_{x}}=-i \partial / \partial x$ and $\widehat{p_{y}}=-i \partial / \partial y$ the momentum operators, and $\hat{I}=|A|^{2}$. The propagation equation (2.1) can be solved using the operator methods of quantum mechanics, i.e., by imposing the following commuting relation between position and momentum operators:

$$
\left[\widehat{x}_{l}, \widehat{p}_{J}\right]=i \delta_{i j}
$$

With this approach, we can derive the root mean square (RMS) width evolution along $\mathrm{z}$ for a centered beam, that reads:

where

$$
\left\langle x^{2}+y^{2}\right\rangle(z)=\frac{\left\langle x^{2}+y^{2}\right\rangle_{0}}{2}\left[(1+C)+(1-C) \cos \cos \left(z \sqrt{\frac{8 \beta}{k_{0}}}\right)\right]
$$

$$
\begin{gathered}
C=\frac{1}{2 \beta} \frac{\left\langle H_{0}\right\rangle}{\left\langle x^{2}+y^{2}\right\rangle_{0}}-1, \\
\left\langle H_{0}\right\rangle=\frac{1}{k_{0}}\left(\left\langle p_{x}^{2}\right\rangle+\left\langle p_{y}^{2}\right\rangle\right)+2 \beta\left(\left\langle x^{2}\right\rangle+\left\langle y^{2}\right\rangle\right)-\gamma\langle I\rangle,
\end{gathered}
$$

and we have imposed $\frac{d\left\langle x^{2}+y^{2}\right\rangle}{d z}=0$ at $z=0$ as the initial condition. These results show that the beam width periodically oscillates during the propagation. Furthermore, there is a critical value of the beam power, above which the amplitude of the oscillation becomes negative, i.e., the beam collapses. Such a threshold, given by the condition $\mathrm{C}=0$, can be estimated to be of a few megawatts for a centered Gaussian beam. Our results are in good agreement with variational approach solutions, that are based on the assumption that the beam keeps its shape (e.g., Gaussian) along all of its propagation [5]. 
Here, we advance the theory of SSI in GRIN MMFs, since the exact solution for the nonlinear dynamics can be computed even for speckled beams. In Fig. 2.1 we plot the oscillating behavior of the RMS width, which is the main property of SSI in GRIN fibers. According to eq. 2.5, the periodicity of SSI only depends on the fiber characteristics (as summarized by the parameter $\beta$ ) and the laser wavelength, but it does not vary with laser power. In the following, we give an experimental confirmation of these model predictions.

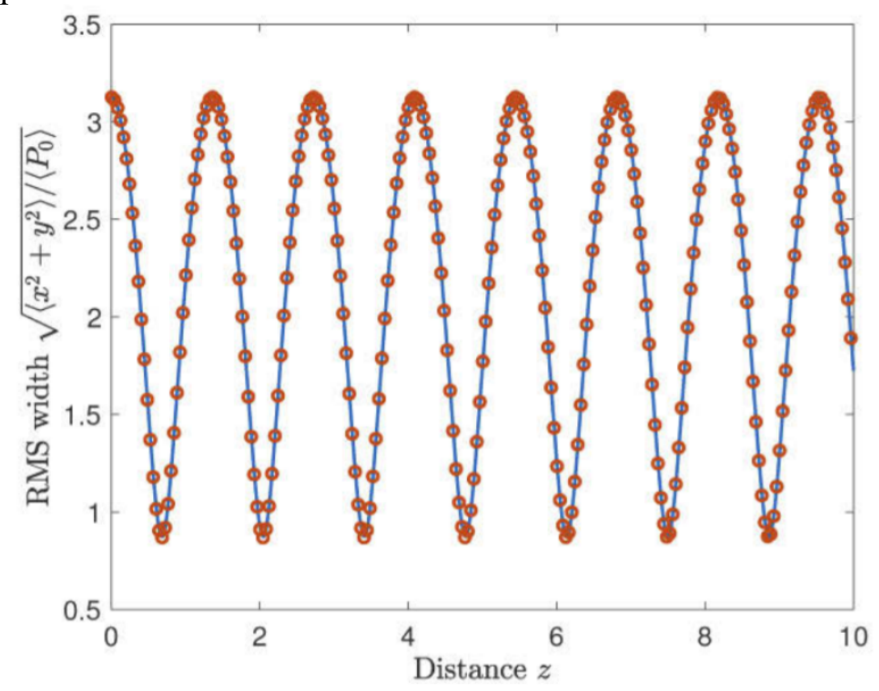

Fig. 2.1 Oscillating behavior of the beam RMS width along propagation distance $\mathrm{z}$.

\subsection{Experiments:}

In our experiments of SSI, we used $174 \mathrm{fs}$ pulses at $\lambda=1030 \mathrm{~nm}$, with $20 \mu \mathrm{m}$ input diameter, coupled to commercially available $4 \mathrm{~cm}$ long GRIN fibers. The SSI of the laser beam inside the fibers can be directly visualized via UL of material defects. These produce a violet light, visible even by the naked eye, when the laser power is sufficiently high (to this purpose we set the highest repetition rate achievable by our laser, i.e., $100 \mathrm{kHz}$ ). By means of a digital microscope (Dinolite-AM3113T) we magnified the resulting spatial emission, could and distinguish well the scattering points periodicity. In Figs. 2.2a and 2.2b we compare images taken for GRIN fibers with different core radius.

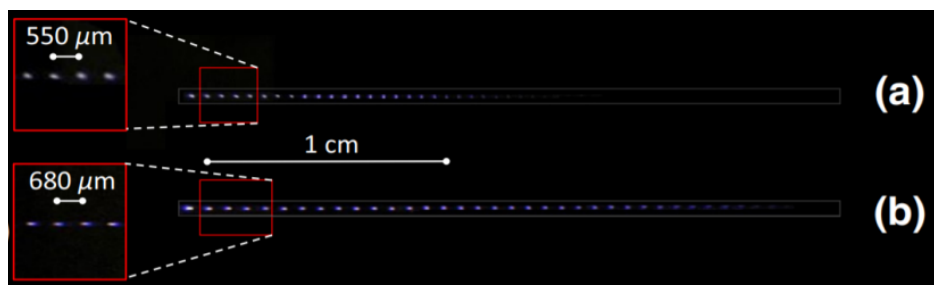

Fig. 2.2 SSI experimental evidence. $(a, b)$ Microscope image of UL scattered out from the fiber side at the maximum intensity points due to SSI for 50/125 (a) and 62.5/125 (b) GRIN fibers; light propagates from the left to the right.

The experimental values of the SSI period (that visibly change in the two cases) are in excellent quantitative agreement with the theoretical values. Furthermore, as expected by the theory, in Fig. 2.3a we show that the SSI period does not vary with input power, at least within experimental measurement errors.

As a last result, we collected side-scattered light by means of a lens into a VIS-IR spectrometer (Avaspec-2048). We show in Fig. $2.3 \mathrm{~b}$ the measured spectrum, when coupling into the GRIN fiber pulses with different peak powers $P_{p}$. Besides the pump at $1030 \mathrm{~nm}$ and the third harmonic generation (THG) contribution at $343 \mathrm{~nm}$, one may notice two main peaks for $P_{p}$ $>1.5 \mathrm{MW}$ : a broader one between $400 \mathrm{~nm}$ and $550 \mathrm{~nm}$, that is mainly given by the Germanium-related Oxygen Deficiency Centers (ODCs); whereas the emission peak around $650 \mathrm{~nm}$ is an intrinsic characteristic of pure silica, and it is due to NonBridging-Oxygen-Hole-Centers (NBOHCs). Let us point out that the Ge-related ODCs contribution is quite higher than that of NBHOCs, because of the extrinsic Ge concentration, which shapes the index profile of the core. 
(a)

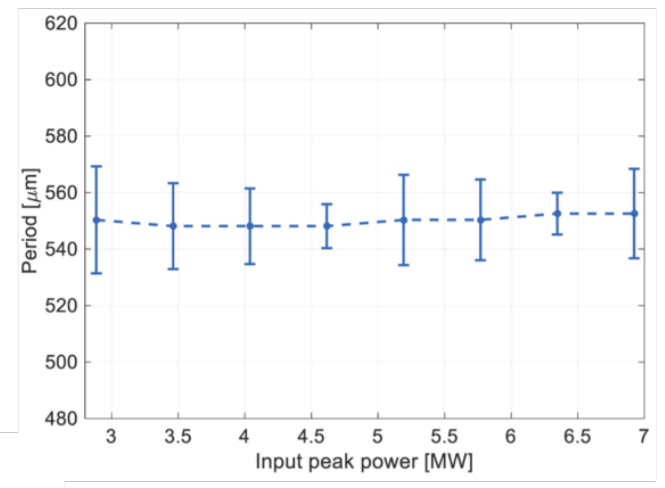

(b)

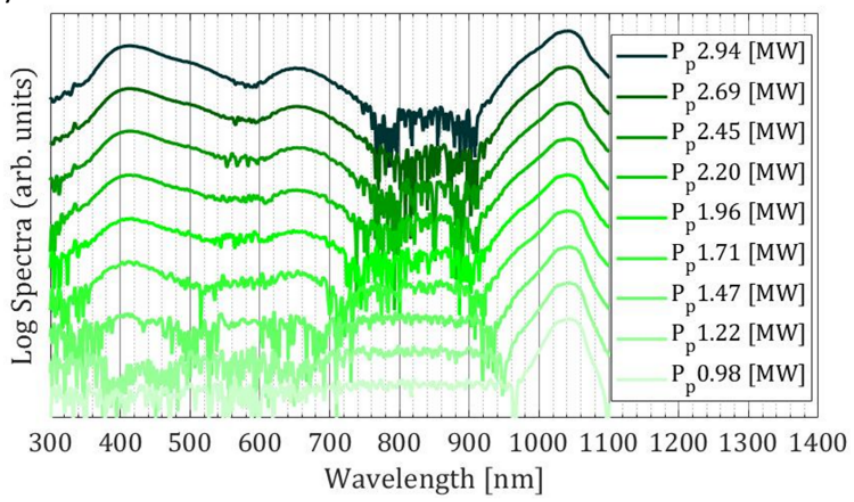

Fig.2.3 Experimental characterization of SSI. (a) Invariance of the SSI period with input laser peak power. (b) Measured UL spectrum in a log-scale, for different input laser peak powers.

The UL of both ODCs and NBOHCs is excited by MPA in the deep ultra-violet range [7]. The presence of MPA processes strongly affect the beam dynamics. One may notice from Fig. 2.2a,b that the UL intensity vanishes after a few centimeters of propagation, meaning that the beam power has been damped. In the next section, we show how the presence of MPA mechanisms affects the fiber-optical transmission.

\section{MULTIPHOTON ABSORPTION IN MM FIBER}

In this section, we experimentally show how the MPA of intense laser pulses affects the dynamics of a beam propagating inside a MMF. In particular, we highlight the role of the SSI in GRIN fibers, and we propose a model involving an average $\mathrm{N}$-photon absorption mechanism in order to describe beam evolution. In spite of its simplicity, this model is able to quantitatively reproduce the experimental transmission drop. Our results show that, differently from the continuous-wave regime, where Brillouin scattering establishes the power transmission limitation, MPA mechanisms turn out to be a relevant nonlinear limitation to the peak power, when high-intensity ultrashort pulses propagate in MMFs.

\subsection{Experiments:}

The experimental set-up is the same as in the previous section, but the length of the 50/125 GRIN fiber is varied. In a cutback experiment, we monitor the pulse energy at the fiber input and output by means of a power meter. The results, reported in Fig.3.1, show a linear dependence of output pulse energy on input pulse energy, when the latter is lower than about $150 \mathrm{~nJ}$. In this region, highlighted in green in the figure, the energy transmission of the fiber is a constant, and we can dub this energy range as the linear transmission regime. In figure 3.1 we traced a dashed black line that fits quite well all of the curves, obtained in this regime for different fiber lengths. This means that energy losses (quantifiable from the slope of the line) in the green area are only due to input coupling conditions (e.g., input position offset, or small tilt angle). Conversely, when the input pulse energy becomes higher than about $150 \mathrm{~nJ}$, the curves spread, stepping away from the dashed line. This is the fingerprint of the nonlinear transmission regime, where the output pulse energy does not linearly scale with the input one anymore. It is worth noting that linear fiber losses are negligible for these fiber lengths, given the linear absorption coefficient of $0.25 \mathrm{~dB} / \mathrm{km}$. 


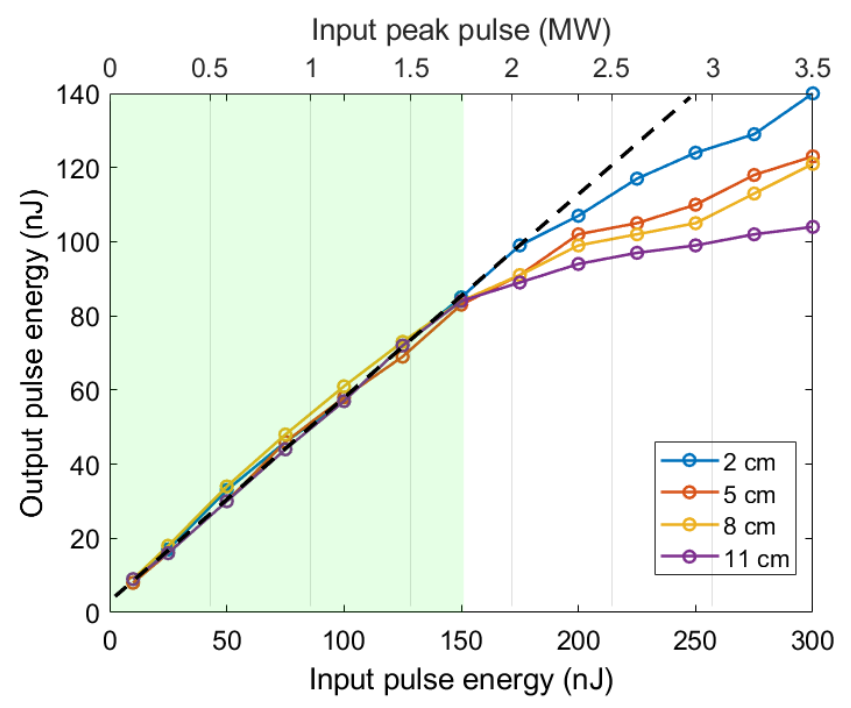

Fig. 3.1 Cutback transmission experiment. The dashed line is the linear fit of all the curves in the linear transmission regime (indicated by the green area).

In order to investigate the nature of nonlinear losses, we monitored the fiber output power when varying the laser repetition rate. The experimental results are shown in Fig. 3.2: a linear trend was observed for peak powers in both the linear and in the nonlinear loss regime. This allows us to claim that the nature of nonlinear losses is purely optical, involving MPA mechanisms, as the one providing defects UL, since we can exclude any thermal effect (phonon excitation) contributions.

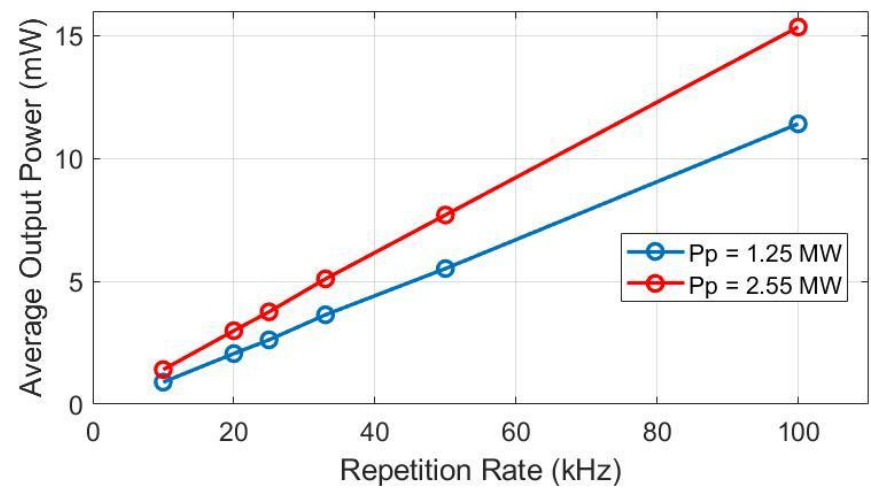

Fig. 3.2 Dependence of average output power upon laser repetition rate, for input peak powers in the linear (1.25 MW) and the nonlinear (2.55 MW) regime, respectively.

Nonlinear transmission losses also occur in Step-Index fibers, in which SSI is absent, and UL only appears in a single spot, the Kerr self-focusing point (see Fig. 3.3a,b). As a result, nonlinear attenuation (defined as the transmission difference between the linear and nonlinear regimes) is much more significant in GRIN fibers for relatively low input pulse energies, when compared to the case of Step-Index fibers, as shown in Fig. 3.3c. This result confirms the role played by SSI in GRIN fibers to enhance nonlinear optical losses: the associated periodic beam shrinking results in an increase of the local beam intensity, making MPA highly significant at multiple points during pulse propagation. 
(a)

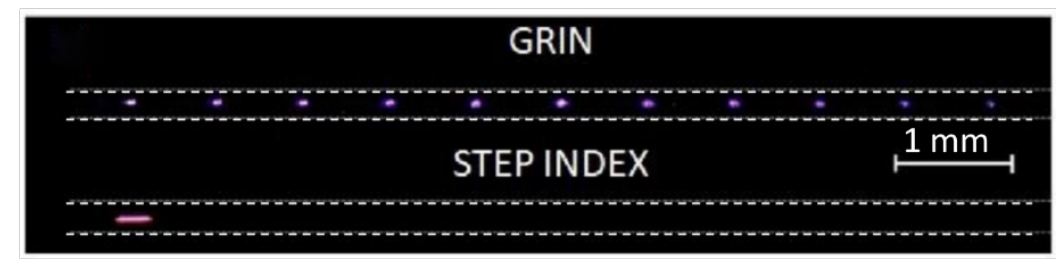

(c)

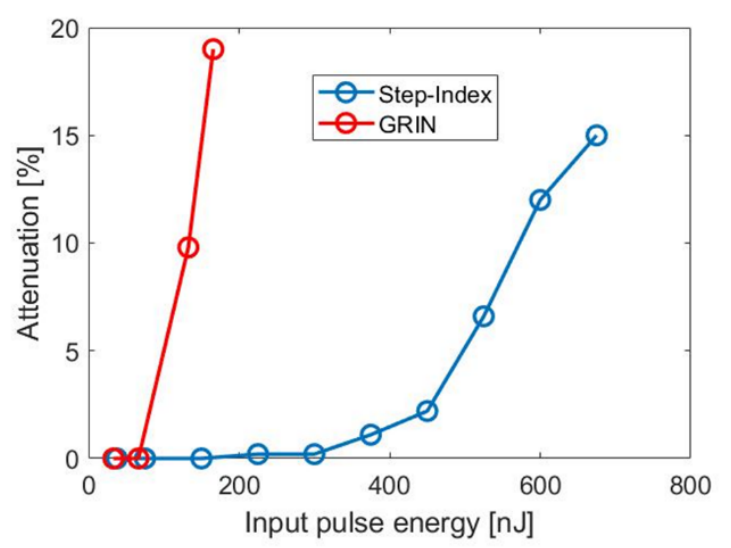

Fig.3.3 Step-Index and GRIN comparison. (a,b) Microscope images of the UL. (c) Nonlinear optical attenuation as a function of input pulse energy.

\subsection{Modelling:}

Although nonlinear losses can be due to several mechanisms, we propose a model in which an effective N-photon absorption term sums up all the nonlinear losses contributions. We add to the propagation equation (2.1) a new term, accounting for MPA

$$
\frac{\partial A}{\partial z}-i \frac{1}{2 k_{0}}\left(\frac{\partial^{2} A}{\partial x^{2}}+\frac{\partial^{2} A}{\partial y^{2}}\right)+i \frac{k_{0} \Delta}{\rho^{2}}\left(x^{2}+y^{2}\right) A=i \frac{k_{0} n_{2}}{n_{c o}}|A|^{2} A-\frac{1}{2} \alpha_{N}|A|^{2 N-2} A .
$$

Here, $N$ represents an average number of photons involved in the absorption process. As in the variational approach, we make the approximation that the beam always keeps a Gaussian shape along its whole propagation. In this way, the evolution of the beam peak power obeys the equation

$$
\frac{d P_{p}}{d z}=-\frac{\alpha_{N}}{2 N-1} \frac{P_{p}^{N}}{\left(\pi a^{2}\right)^{N-1}}
$$

where $a$ is the beam waist. The MPA contribution leads to nearly step-wise transmission drops at points of minimum beam diameter, which oscillates because of SSI. These points correspond to local maxima of the beam intensity, as shown in Fig. 3.4a.

As a further approximation, we consider $a$ being independent of the beam power, so that we can fit all curves in Fig. 3.1 with the same parameters. The good agreement found between numerical and experimental values of the transmission is reported in Fig. 3.4b. The fit returns an average number of photons $N=2.68$, while five photons are involved in the UL [7]. This evidence demonstrates that more than one process is responsible for MPA. In particular, a value of $N$ smaller than 3 indicates that the contribution of two-photon absorption processes is relevant, but not sufficient to fully describe the observed beam dynamics. 
(a)

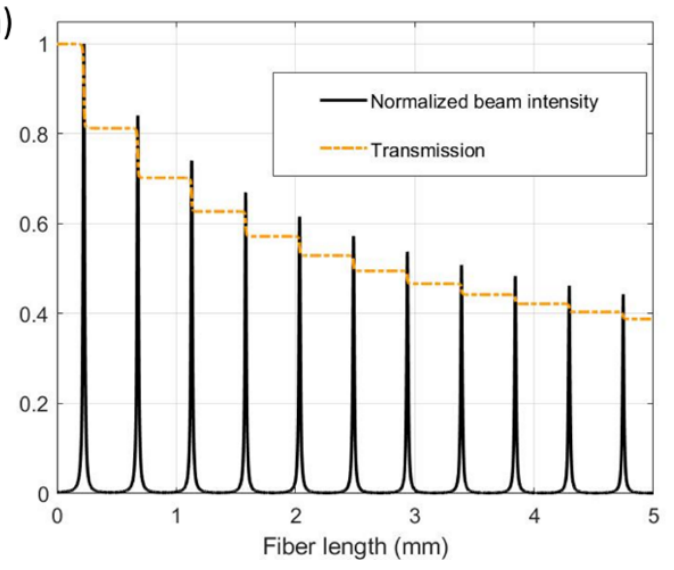

(b)

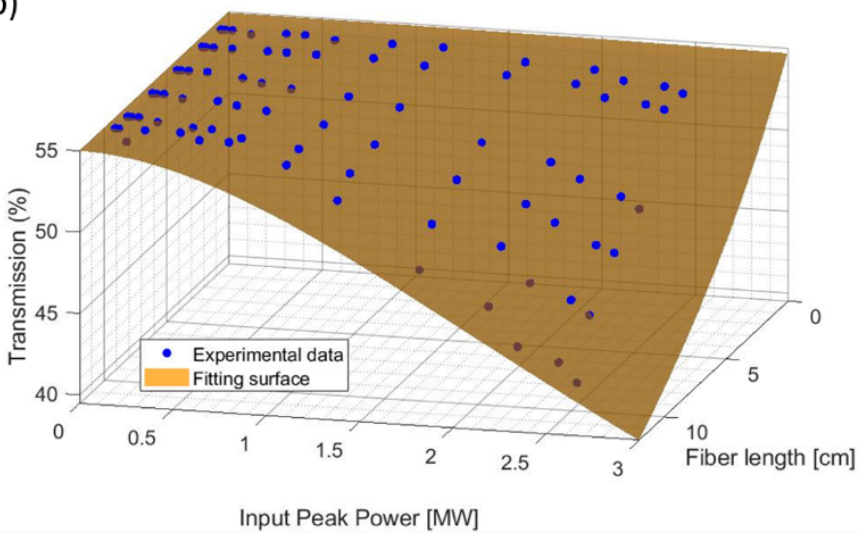

Fig. 3.4 N-photon absorption modelling. (a) Beam intensity (normalized to its maximum value) and transmission trends along the first $5 \mathrm{~mm}$ of propagation. (b) Fit of the transmission experimental data in Fig. 3.1.

We repeated our measurements in the anomalous dispersion regime, at telecom wavelengths. Even in this regime the appearance of UL at the SSI points and optical nonlinear losses were observed. In the next section, we will show how this phenomenology strongly affects the dynamics of Raman solitons arising from the fission of high-energy femtosecond input pulses.

\section{HIGH ENERGY PULSE FISSION IN MM FIBER}

In order to better understand the temporal dynamics of pulses propagating in the high-power regime, we experimentally studied the fission at high input energies (up to $550 \mathrm{~nJ}$, MW peak powers) of high-order MM solitons in a GRIN fiber. When high energy, ultra-short pulses are injected in the GRIN fiber, a novel regime is observed, where spatiotemporal solitons produced by the fission have a nearly constant Raman wavelength shift, and the same pulse width over a wide range of soliton energies.

Our experiments permit to describe the range of validity of a reduced singlemode (SM) soliton description $[5,8,9]$. We find that high energy solitons originating from the fission process differ from the SM description, showing different energies, but comparable pulse widths, and clamped, but different wavelengths. Multimode pulse fission at MW peak powers produces a train of fundamental spatiotemporal solitons, which tend to share the same pulse width. Even when composed by several non-degenerate modes, fundamental solitons must fulfil the condition

$$
N^{2}=\gamma_{j} P_{j} \frac{T_{0 j}{ }^{2}}{\left|\beta_{2 j}\right|=1},
$$

where, for the $j$-th soliton, $\gamma_{j}$ is the nonlinear coefficient, $P_{j}$ the peak power, $T_{0 j}$ the pulse width (over 1.763), and $\beta_{2 j}$ the dispersion, respectively. All parameters are evaluated at the specific wavelength of each soliton; $\gamma_{j}$ must account for an effective beam waist $w_{j}$, reduced with respect to the input as a consequence of periodic beam self-imaging. In order to reach the fundamental soliton condition, pulses with different peak powers red-shift their wavelength under the action of Raman nonlinearity and high-order dispersion; the following condition holds for each soliton

$$
T_{0 j}=\frac{\lambda_{j}\left|\beta_{2 j}\right| w_{j}^{2}}{n_{2} E_{S, j}},
$$

with $E_{s, j}$ the soliton energy; the pulse width must therefore increase, in accordance with the experienced dispersion and wavelength up-shift. The Raman wavelength shift resulting from the fission process is much faster than the value predicted by the single-mode soliton perturbation formula 


$$
\lambda_{j}(z)=\lambda_{f, j}+\frac{4 \lambda_{f, j} n_{2} T_{R} E_{s, j}}{15 \pi c w_{j}^{2} T_{0 j}^{3}}\left(z-z_{f}\right)
$$

After the initial soliton wavelength multiplex is formed, solitons tend to conserve the initial common pulse width upon propagation, with different peak powers and effective waists. Wavelengths start shifting with slower speed during a second propagation phase, this time according to Eq. 4.3.

As illustrated in Fig.4.1, MM soliton fission dynamics in GRIN multimode fibers was experimentally studied by launching high energy $130 \mathrm{fs}$ pulses at $\lambda=1550 \mathrm{~nm}$, with $20 \mu \mathrm{m}$ diameter, into a GRIN fiber $(50 \mu \mathrm{m}$ core, $125 \mu \mathrm{m}$ cladding, relative index difference $\Delta=0.0103$, length $30 \mathrm{~cm}$ ). At the output, spectra were observed between 300 and $3000 \mathrm{~nm}$ by spectrum analyzers (Fastlite Mozza, Yokogawa AQ6370D); pulses were measured by autocorrelation (APE pulseCheck 50), the near-field was observed by an InGaAs camera (Hamamatsu C12741-03).

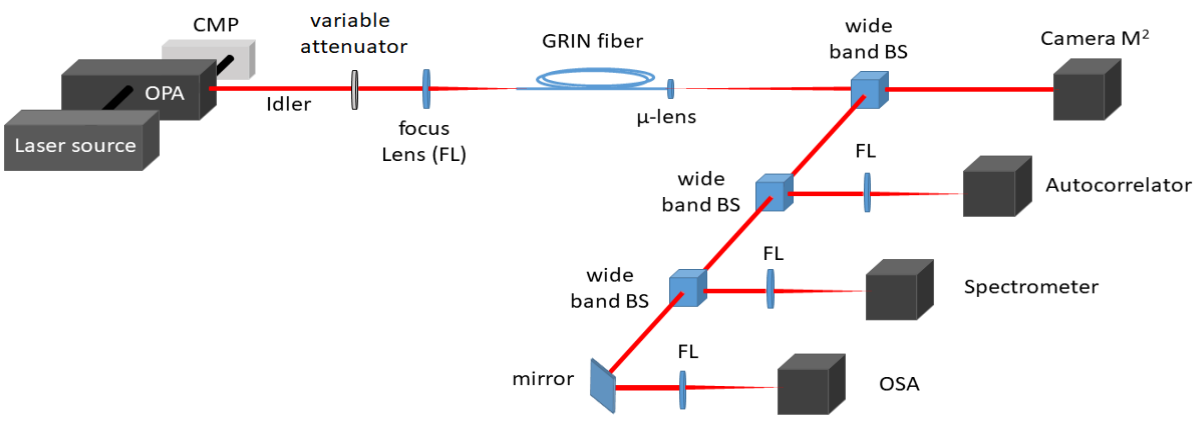

Figure 4.1. The experimental setup.
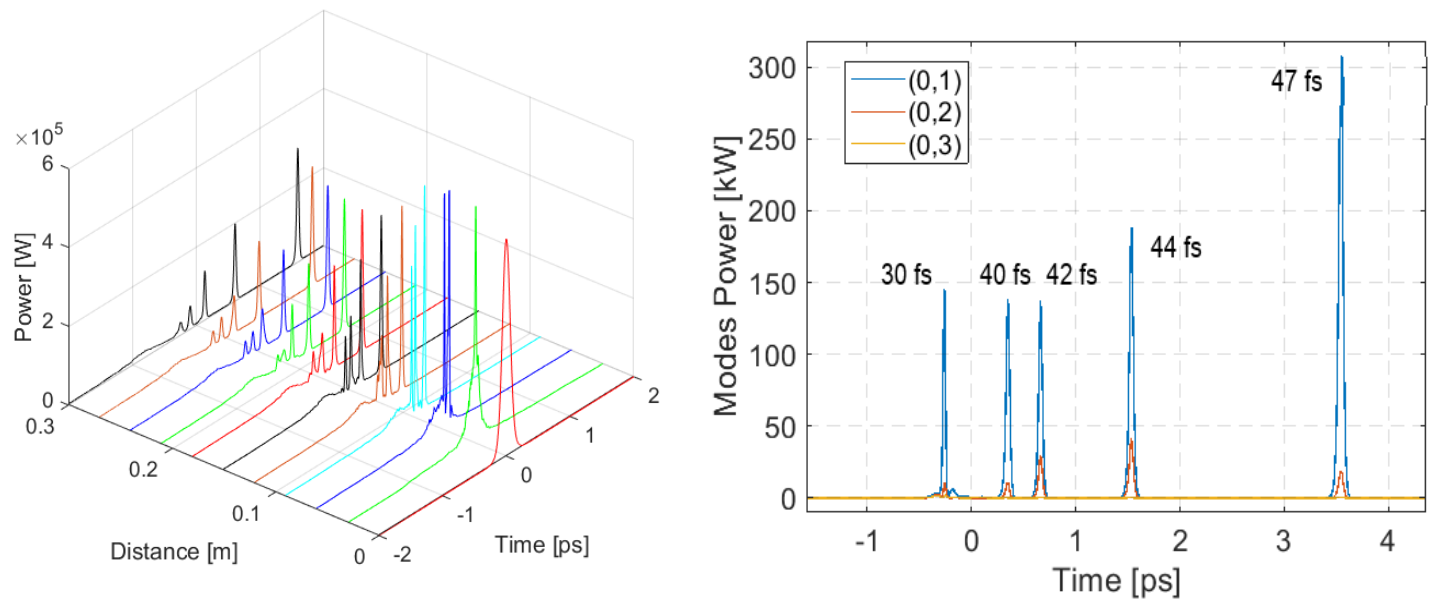

Figure 4.2. Numerical simulation of multimode soliton fission.

Pulse dynamics was also numerically investigated by means of a vectorial 3D+1 model, accounting for Kerr and Raman nonlinearity, dispersion up to 4-th order, waveguide term with parabolic index profile $n(x, y)$ and cladding index $n_{\text {clad }}$, wavelength dependent losses, nonlinear losses, and third harmonic generation (THG). Numerical simulations (Fig. 4.2 left) show that the input pulse experiences fission in the first $10 \mathrm{~cm}$ of fiber. A train of MM solitons is produced, multiplexed at different wavelengths in the first $10 \mathrm{~cm}$. Once generated, solitons suffer red-shifting by stimulated Raman scattering (SRS). Pulse intensity exhibits fast oscillations $(0.55 \mathrm{~mm}$ period) owing to SSI, and long-range peaks $(0$ to $10 \mathrm{~cm})$ at points of soliton fission. 
In the example of Fig. 4.2, three nondegenerate modes (LP01, $\mathrm{LP}_{02}$, and $\left.\mathrm{LP}_{03}\right)$ are propagated over $30 \mathrm{~cm}$ of GRIN fiber. At high powers, the initial pulse splits into multiple spatiotemporal solitons; for each soliton, the three nondegenerate modes are trapped in time and self-confined. The output pulse widths (Fig. 4.2 right) are similar (30-47 fs) for all solitons, in spite of their different energies.

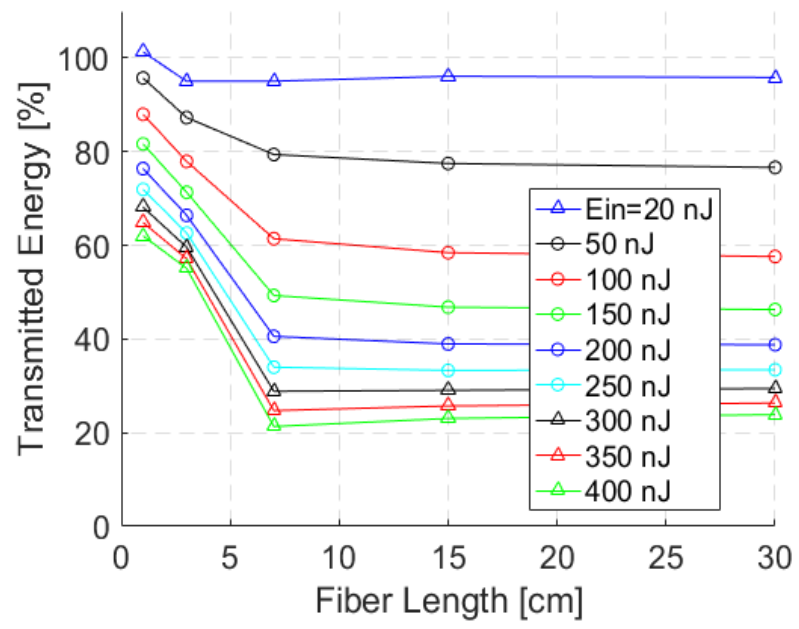

Fig. 4.3. Measured transmitted energy for different lengths of fiber, and several values of pulse input energy.

Experimental results (see Fig. 4.3) show that the output energy, after $30 \mathrm{~cm}$ of fiber, clamps for input energy $E_{\text {in }}>100 \mathrm{~nJ}$. Energy at wavelength below $1100 \mathrm{~nm}$ represents $10 \%$ of the total. From a cutback experiment, two-photon absorption (TPA) is observed during the pulse fission phase (first $7 \mathrm{~cm}$ of fiber). MPA and scattering of broadband fluorescence are responsible for losses up to $80 \%$, for increasing input pulse energies.
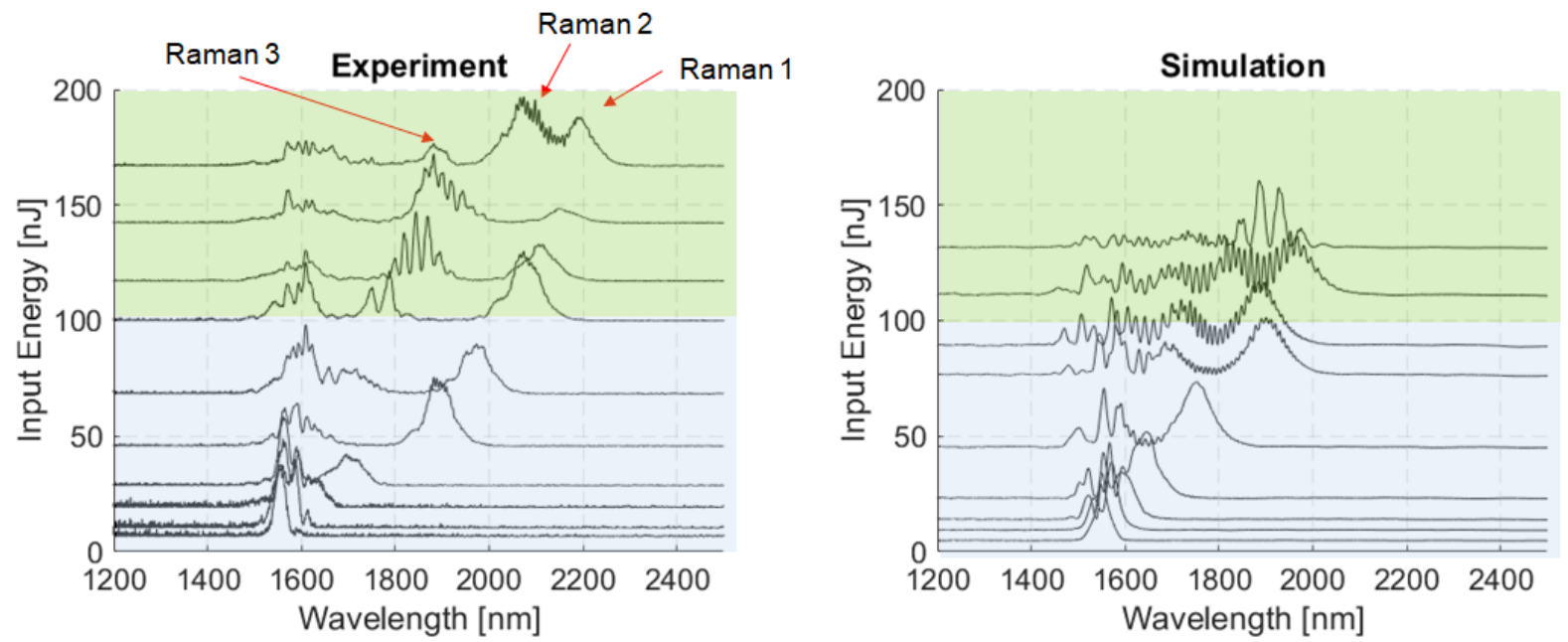

Fig. 4.4. Measured (left) and simulated (right) output spectra, for different input pulse energies.

In Fig. 4.4, Experimental (left) and simulated (right) spectra at different input energies $E_{\text {in }}$ show that the Raman wavelength shift clamps, as a consequence of the presence of nonlinear losses. For increasing $E_{i n}$, progressively larger numbers of MM Raman solitons are generated. Individual MM solitons undergo different amounts of soliton self-frequency shift (SSFS), clamped to 2250-2300 nm. We define Raman 1, Raman 2, Raman 3, the 3 Raman-shifted solitons caused by the fission for increasing energy. Therefore, we may recognize the presence of two different regimes, a low-loss, or linear loss regime, and a high-loss, or nonlinear loss regime, where a large part of the input pulse energy is absorbed by MPA. 
In Fig. 4.5, we compare experimental data (empty squares and empty circles) with numerical simulations (solid curves) of the wavelength of solitons Raman 1 and Raman 2, respectively, as a function of their output energy, $E_{s}$ (energy is not that of the input pulse this time). Up to $E_{s}=15 \mathrm{~nJ}$, the soliton wavelength increases with soliton energy, according to the analytical single-mode SSFS Eq. 4.3 (dashed line). For $5<E_{s}<15 \mathrm{~nJ}$ (linear loss regime), the soliton wavelength still increases, but with a higher slope. For $E_{s}>15 \mathrm{~nJ}$ (nonlinear loss regime), experimental data show an irregular distribution of Raman soliton wavelengths, oscillating around $2100 \mathrm{~nm}$ for the Raman 1 soliton, and $1900 \mathrm{~nm}$ for the Raman 2 soliton. In this regime, soliton energies may reach $50 \mathrm{~nJ}$.

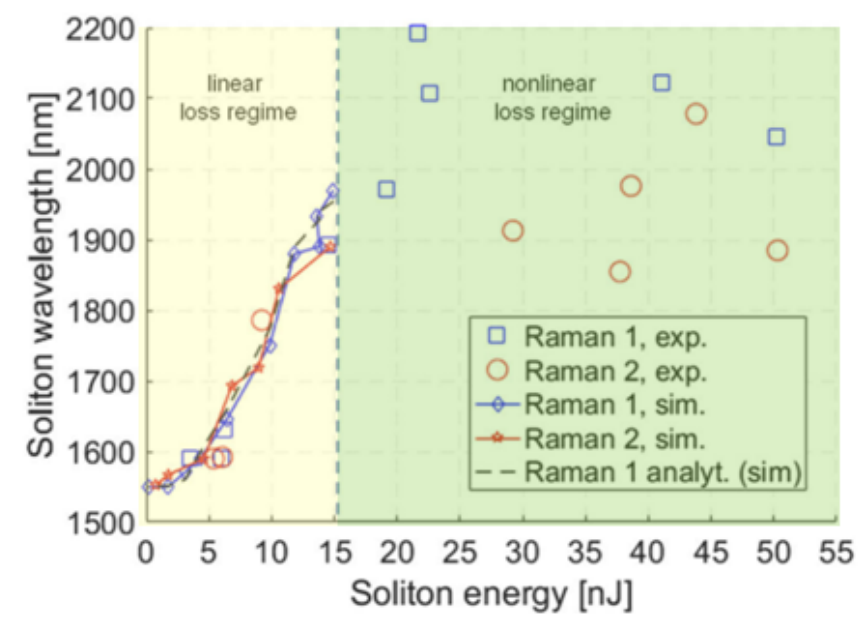

Fig. 4.5. Output soliton wavelength vs. soliton energy.

In Fig. 4.6 we illustrate the output solitons pulsewidth vs. soliton energy: we may note that, for soliton energies $E_{s}<15 \mathrm{~nJ}$ (linear-loss), the pulse width of individual Raman MM solitons is well approximated by the single-mode (SM) analytical soliton formula, Eq. 4.2. A train of pulses with sech temporal shape, different peak powers, and wavelengths, but nearly equal pulse widths (around $50 \mathrm{fs}$ ) is generated for soliton energies $E_{s}>5 \mathrm{~nJ}$, in good agreement with numerical simulations.

For $E_{s}>15 \mathrm{~nJ}$ (nonlinear loss regime), Eq. 4.2 for the soliton pulse width (black crosses) predicts a narrower pulse width, when compared with the experimentally observed soliton pulse durations. Experimental data show that all MM Ramanshifted solitons possess different wavelengths and energies, but conserve nearly the same and constant pulse width (with respect to soliton energy), with a value between $40 \mathrm{fs}$ and $60 \mathrm{fs}$.

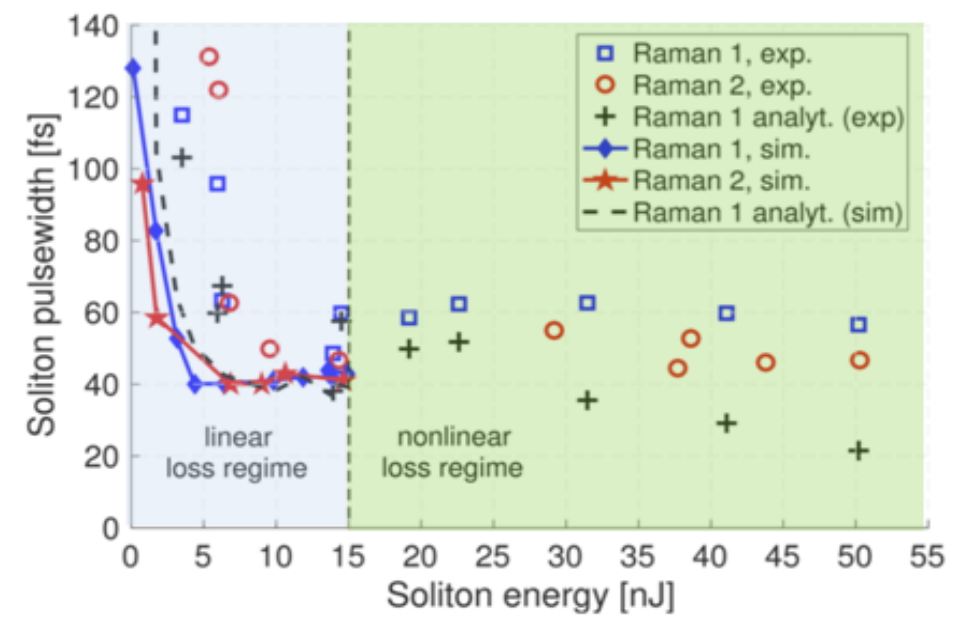

Fig. 4.6. Output soliton pulse width vs. soliton energy. 


\section{SOLITON EVOLUTION IN MM FIBER}

Further experimental investigations of MM soliton evolutions have been conducted over relatively long spans (e.g., 100 $\mathrm{m}$ ) of GRIN fiber. Results were quite surprising, as they indicate that MM solitons originating from the temporal trapping of several nondegenerate modes, tend to evolve into singlemode solitons, provided the initial nonlinear length is comparable with the modal walk-off length, and the propagation evolves over hundreds of nonlinearity lengths.

Fig. 5.1 compares experimental and simulation results for input pulses at $1550 \mathrm{~nm}$, with input beam diameter of approximately $30 \mu \mathrm{m}$, suitable for exciting the first 3 axial modes $\mathrm{LP}_{01}, \mathrm{LP}_{02}, \mathrm{LP}_{03}$. The soliton pulse width, as measured by autocorrelations, is provided vs. input peak power. In the figure, a minimum pulse width of 260 fs is reached for the input energy of $2 \mathrm{~nJ}$; this pulse duration is considerably larger than what was observed after $30 \mathrm{~cm}$ (Fig. 4.6), but, as already said for Eq. 4.2, the soliton pulse width must increase with distance as the wavelength and the experienced chromatic dispersion both increase in absolute value.
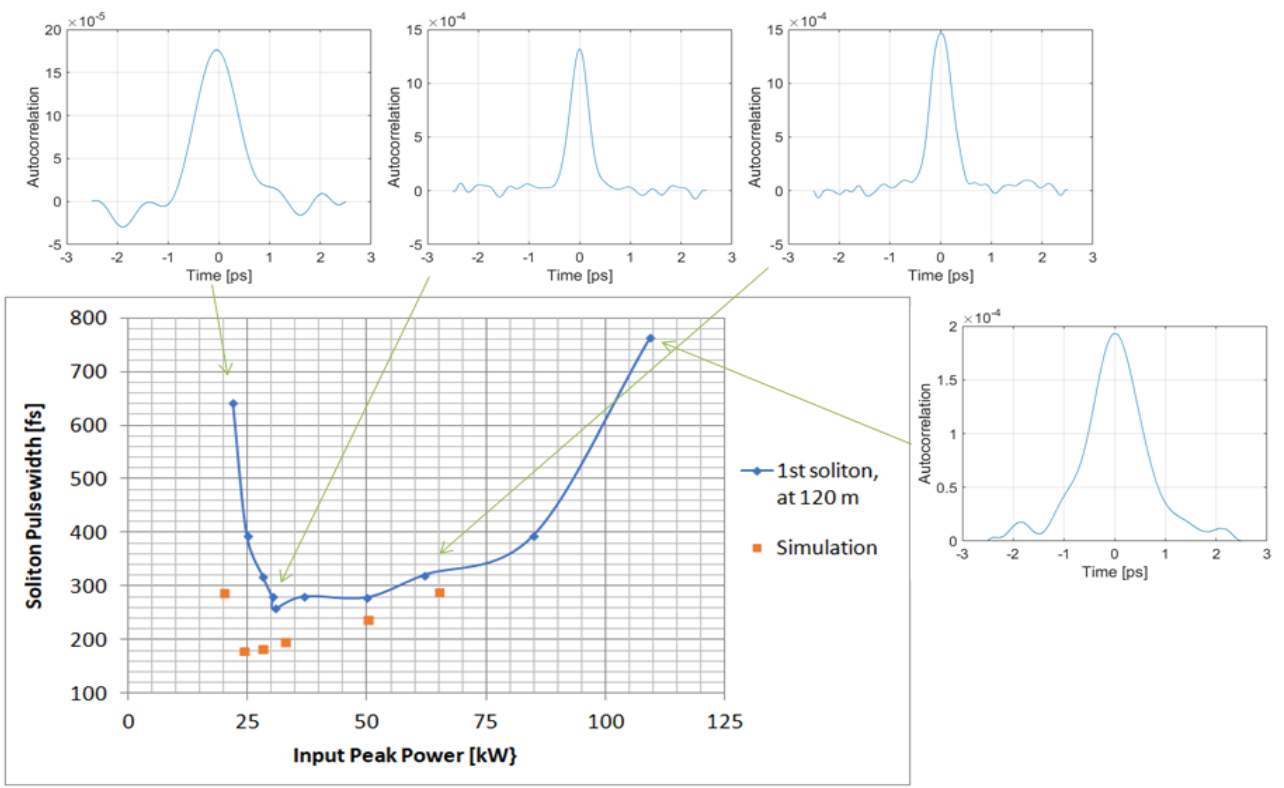

Fig. 5.1. Measured autocorrelations after $100 \mathrm{~m}$ of GRIN fiber.

Fig. 5.2 reports the measured waist of the output near-field. A huge reduction of the beam waist is observed for input peak powers corresponding to the generation of the first and the second soliton, respectively. In the first case, the waist reduces to $8.5 \mu \mathrm{m}$, close to the $7.7 \mu \mathrm{m}$ value of the GRIN fiber's fundamental mode.

The obtained results are confirmed by numerical simulations, which indicate the occurrence of a net transfer of energy from higher-order modes to the $\mathrm{LP}_{01}$ mode, thus acting as an attractor through the process of modal four-wave mixing (FWM). Once formed, the single mode soliton is able to propagate stably in the GRIN fiber.

When the first soliton shifts up to $2000 \mathrm{~nm}$, it is absorbed by the fiber. At higher input energies, multiple solitons are generated; if the first is absorbed, the second is observed at the output, with a behavior which is similar to the first one. 

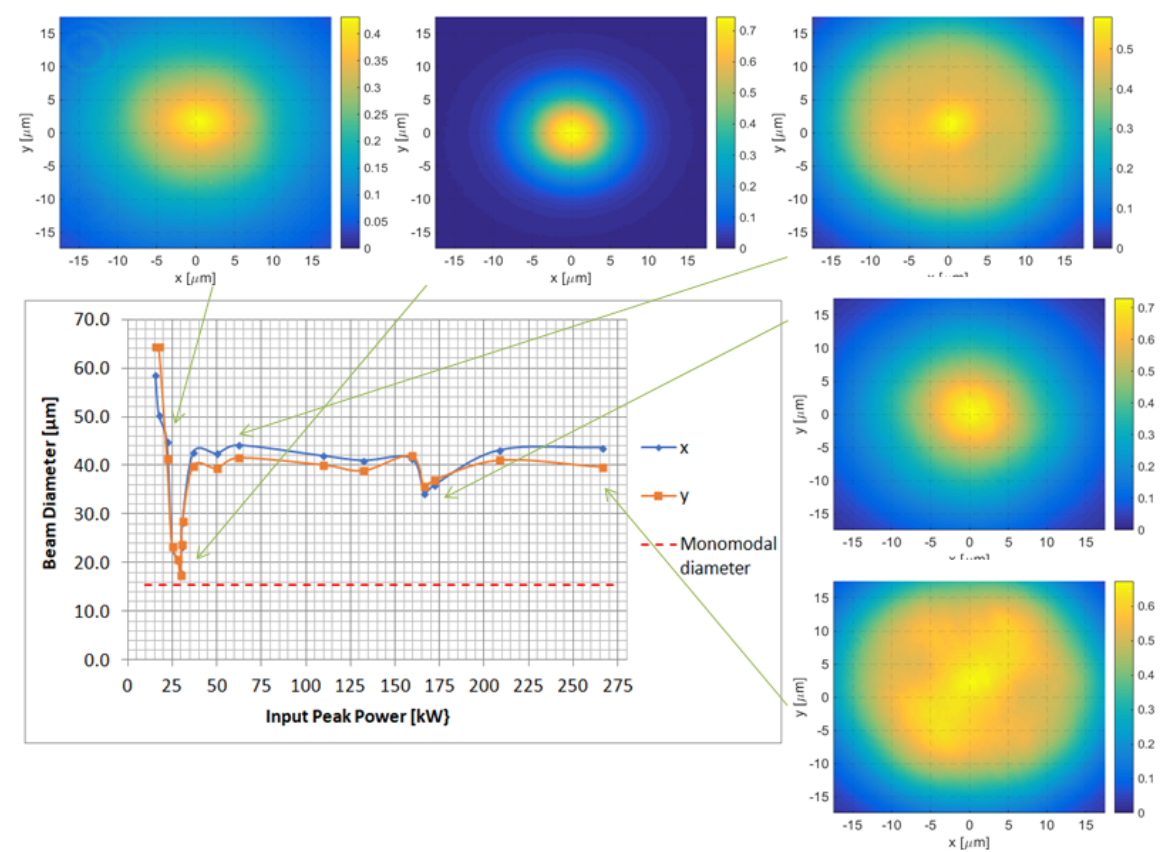

Fig. 5.2. Measured beam waist after $100 \mathrm{~m}$ of GRIN fiber.

\section{CONCLUSIONS}

In this work, we proposed a general equation describing sinusoidal spatial self-imaging (SSI) of light beams propagating in nonlinear GRIN MMFs. This equation predicts the occurrence of periodic along the distance beam waist SSI oscillations with a power-dependent amplitude: the period of these oscillations remains independent of power. This equation holds for any beam shape, that is even for speckled beams. The property of constant SSI period with respect to the pulse power was confirmed experimentally, by observing the up-conversion luminescence that is scattered from the fiber side.

A theory for MPA in MMFs was also introduced, where an effective N-photon absorption term sums up all of the nonlinear loss contributions. Experiments confirmed the theory, by measuring nonlinear losses in MM step-index as well as in GRIN MMFs, as well as observing the up-conversion luminescence at points of maximum beam intensity.

Our experiments demonstrated that high-energy, ultra-short high-order soliton pulse fission in a parabolic GRIN fiber produces trains of wavelength-multiplexed MM fundamental solitons with nearly equal temporal durations, and different energies. The wavelength shift produced by the initial fission process largely dominates the propagation SSFS. In the "linear-loss" regime, which occurs when the generated solitons possess energies below 15 nJ, the pulse width of an individual MM Raman soliton scales with its energy, according to the well-known law for fundamental SM solitons.

For energies around $2 \mathrm{~nJ}$ and ultra-short input pulses, the generation and stable propagation of spatiotemporal solitons was observed over relatively long GRIN fiber distances. For an optimal input pulse energy, the output beam approaches the fundamental mode of the fiber. This was observed both with degenerate and non-degenerate modes at the input.

These results are relevant for advancing the understanding of complex wave propagation phenomena in nonlinear multimode optical fibers, and for using these fibers in the next-generation of optical communication and laser technologies. 


\section{Acknowledgments}

We acknowledge the financial support from the European Union's Horizon 2020 Research and Innovation Programme via the European Research Council (Grants No. 874596 and No. 740355), the Italian Ministry of University and Research (Grant No. R18SPB8227), and the Russian Ministry of Science and Education (Grant No. 14.Y26.31.0017)

\section{REFERENCES}

[1] Renninger, W. H., and Wise, F. H., "Optical solitons in graded-index multimode fibres," Nat. Commun. 4, 1719 (2013).

[2] Krupa, K., Tonello, A., Barthélémy, A., Mansuryan, T., Couderc, V., Millot, G., Modotto, D., Babin, S. A., and Wabnitz, s., "Multimode nonlinear fiber optics, a spatiotemporal avenue," APL Photonics 11, 110901 (2019).

[3] Zitelli, M., Mangini, F., Ferraro, M., Niang, A., Kharenko, D., and Wabnitz, s., "High-energy soliton fission dynamics in multimode GRIN fiber," Optic Express 28(14), 20473-20488 (2020).

[4] Hansson, T., Tonello, A., Mansuryan, T., Mangini, F., Zitelli, M., Ferraro, M., Niang, A., Crescenzi, R., Wabnitz, S., and Couderc, V., "Nonlinear beam self-imaging and self-focusing dynamics in a GRIN multimode optical fiber: theory and experiments," Optic Express 28(16), 24005-24021 (2020).

[5] Karlsson, M., Anderson, D., and Desaix, M., "Dynamics of self-focusing and self-phase modulation in a parabolic index optical fiber," Opt. Lett. 17(1), 22-24 (1992).

[6] Girard, S., Alessi, A., Richard, N., Martin-Samos, L., De Michele, V., Giacomazzi, L., ... and Ouerdane, Y., "Overview of radiation induced point defects in silica-based optical fibers," Reviews in Physics, 4, 100032 (2019).

[7] Mangini, F., Ferraro, M., Zitelli, M., Niang, A., Tonello, A., Couderc, V., and Wabnitz, S., "MultiphotonAbsorption-Excited Up-Conversion Luminescence in Optical Fibers," Physical Review Applied, 14(5), 054063 (2020).

[8] Ahsan, A. S., and Agrawal, G. P., "Spatio-temporal enhancement of raman-induced frequency shifts in gradedindex multimode fibers," Opt. Lett. 44(11), 2637-2640 (2019).

[9] Zitelli, M., Ferraro, M. Mangini F., and Wabnitz, S., "Managing Self-Phase Modulation in Pseudo-linear Multimodal and Monomodal Systems," Journal of Lightwave Technology, doi: 10.1109/JLT.2020.3047155. 